\title{
Grand multiparity as a predictor of adverse pregnancy outcome among women who delivered at a tertiary hospital in Northern Tanzania
}

Zainab Muniro ${ }^{1,2^{*}}$, Clifford Silver Tarimo ${ }^{3}$, Michael J. Mahande², Eusebious Maro ${ }^{1}$ and Bariki Mchome ${ }^{1}$

\begin{abstract}
Background: Grand multiparity has been associated with increased risks of adverse pregnancy outcomes such as post-partum hemorrhage,gestational hypertension, gestationaldiabetes mellitus and high perinatal mortality. There is limited information about the impact of high parity on pregnancy outcomes in Tanzania. This study aimed to determine prevalence, trend and associated adverse pregnancy outcomes of grand multiparity in a tertiary hospital in Northern Tanzania.

Methods: A retrospective cross-sectional study was conducted at Kilimanjaro Christian Medical Centre (KCMC) using maternally linked data from medical birth registry. Women with singleton deliveries from 2006 to 2014 were analyzed. The prevalence of grand-multiparity was computed as proportion to estimate the trend over years. Adverse pregnancy outcomes associated with grand multiparity were estimated using multivariable logistic regression models. A $p$-value of $<0.05$ was considered statistically significant.

Results: The overall prevalence of grand multiparity was 9.44\% ranging from 9.72\% in 2006 to $8.49 \%$ in 2014 . The grand multiparous women had increased odds of prelabour rupture of membranes (Adjusted odds ratio [AOR] 1.78: 95\% Cl:1.28-2.49), stillbirth (AOR 1.66: 95\% Cl:1.31-2.11) and preterm birth delivery (AOR 1.28; 95\% Cl: 1.05-1.56) as compared to women in the lower parity group.

Conclusions: The prevalence of grand multiparity among women in North-Tanzania was 9.44\%. It was significantly associated with adverse pregnancy outcomes. This calls for a need to increase community awareness on its risks, encourage birth control among older women. Delivery-care facilities should prepare for emergency situation when attending deliveries of high parity group.
\end{abstract}

Keywords: Prevalence, Grand multiparity, Low parity, Pregnancy outcomes, North-Tanzania

\section{Background}

As Sustainable Development Goal's (SDG) efforts to address promotion of maternal health and reduction of child mortality becomes intensified, regional specific investigation to inform the public health on potential effect of factors that presents a significant risk to the pregnant mother and the birth outcome are imperative.

\footnotetext{
*Correspondence: zkapemba@gmail.com

'Department of Obstetrics and Gynecology, Kilimanjaro Christian Medical Centre, Box 3010, Moshi, Tanzania

${ }^{2}$ Department of Epidemiology and Biostatistics, Institute of Public Health, Kilimanjaro Christian Medical University College, Box 2240, Moshi, Tanzania Full list of author information is available at the end of the article
}

Huge disparity in the fertility rate exist between the developed and developing countries. Low-and-middle income countries including Tanzania's fertility rate have still relatively high annual fertility rate as compared to high income countries [1]. This trend may pose substantial risk to the pregnant mother and result in adverse birth outcome. Among other factors, grand multiparity is hypothesized to play a significant role in adverse maternal outcomes especially in low-and-middle income countries like Tanzania.

Grand-multiparity (GMP) condition has been defined differently in several literature [1-3]. Some literature

(c) The Author(s). 2019 Open Access This article is distributed under the terms of the Creative Commons Attribution 4.0 International License (http://creativecommons.org/licenses/by/4.0/), which permits unrestricted use, distribution, and reproduction in any medium, provided you give appropriate credit to the original author(s) and the source, provide a link to the Creative Commons license, and indicate if changes were made. The Creative Commons Public Domain Dedication waiver (http://creativecommons.org/publicdomain/zero/1.0/) applies to the data made available in this article, unless otherwise stated. 
define it as a woman who delivered four or more times while others consider of six or more deliveries [4]. Furthermore, the International Federation of Gynecology and Obstetrics defined GMP as deliveries of a fifth to ninth, while naming those undergoing their tenth or more deliveries as great grand-multiparous or huge great grand-multiparous [5-8]. According to the CDC report of 2004, the prevalence of grand multiparity was reported as low as 3-4\% in developed countries as compared to $19.3 \%$ in developing countries, $[9,10]$. The lower prevalence in developed countries has been attributed to high use of modern family planning and optimal obstetric care while the high prevalence of GMP in low income countries is fueled by gender desirability, low education and desire for more offspring to have large family size [11, 12]. Adverse outcomes associated with GMP include diabetes, premature labor, maternal and perinatal mortality, placenta previa, genital sepsis, postpartum hemorrhage ( $\mathrm{PPH})$, utero-vaginal prolapse, hypertension and Intrauterine fetal death (IUFD) [6, 7, $10,13]$. Controversy prevails in the effect of high parity on these complications since some other studies report no increased incidences of obstetric complications [1, 14]. Despite efforts to reduce maternal and perinatal adverse outcomes in Tanzania, maternal morbidities and mortalities are high. This study takes advantage of existing large scale birth registry data to reaffirm existing small scale evidence conducted in 2013 in one of the tertiary facility in Tanzania and attempt to account for change in magnitude of grandmultiparity overtime with an additional capability to capture infrequent adverse outcome. Moreover, describing a trend of grand multiparity overtime may provide a reflection of effectiveness of contraceptive coverage in this population. This study's primary aim was to determine the association between GMP and adverse pregnancy outcome. Moreover, we aimed at assessing the prevalence, trend and associated pregnancy outcomes of grand multiparity in a tertiary hospital in Northern Tanzania over nine (9) years.

\section{Methods}

Study design, setting \& population

This was a cross-sectional study using maternally linked data from Kilimanjaro Christian Medical Centre (KCMC) medical birth registry. KCMC is a referral consultants and teaching hospital located in Moshi, Kilimanjaro region in Northern-Tanzania. According to the Tanzania National Census of 2012, the population of Kilimanjaro region is estimated to be 1.6 million people. The average deliveries per year is around 4000. The KCMC medical birth registry was established in the year 1999 as pilot and officially started to operate in 2000. Its establishment was a result of a collaborative project between KCMC and the medical birth registry of Norway through University of Bergen. Since then, medical records for all women who deliver at KCMC and their newborns have been prospectively collected and stored at the medical birth registry. In this study, multiparous women (parity of 2 or more) of 28 weeks of gestation or more, with singleton births from the year 2006 to 2014 were studied. We excluded multiple pregnancy to avoid overrepresentation of high-risk women which might exaggerate the pregnancy outcomes. The final sample analyzed comprised of 18,441 deliveries (Fig. 1).

\section{Data collection methods and tools}

Socio-demographic and obstetric information for all women who delivered at KCMC were recorded at the

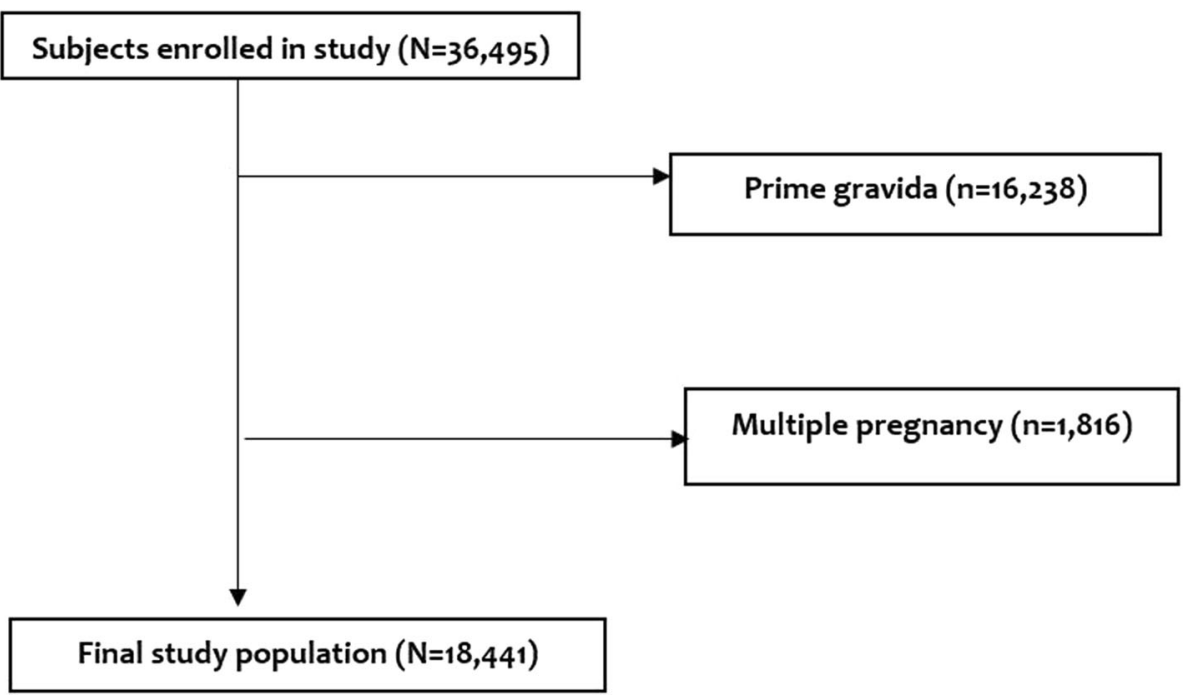

Fig. 1 Schematic Diagram of the selected study participants 


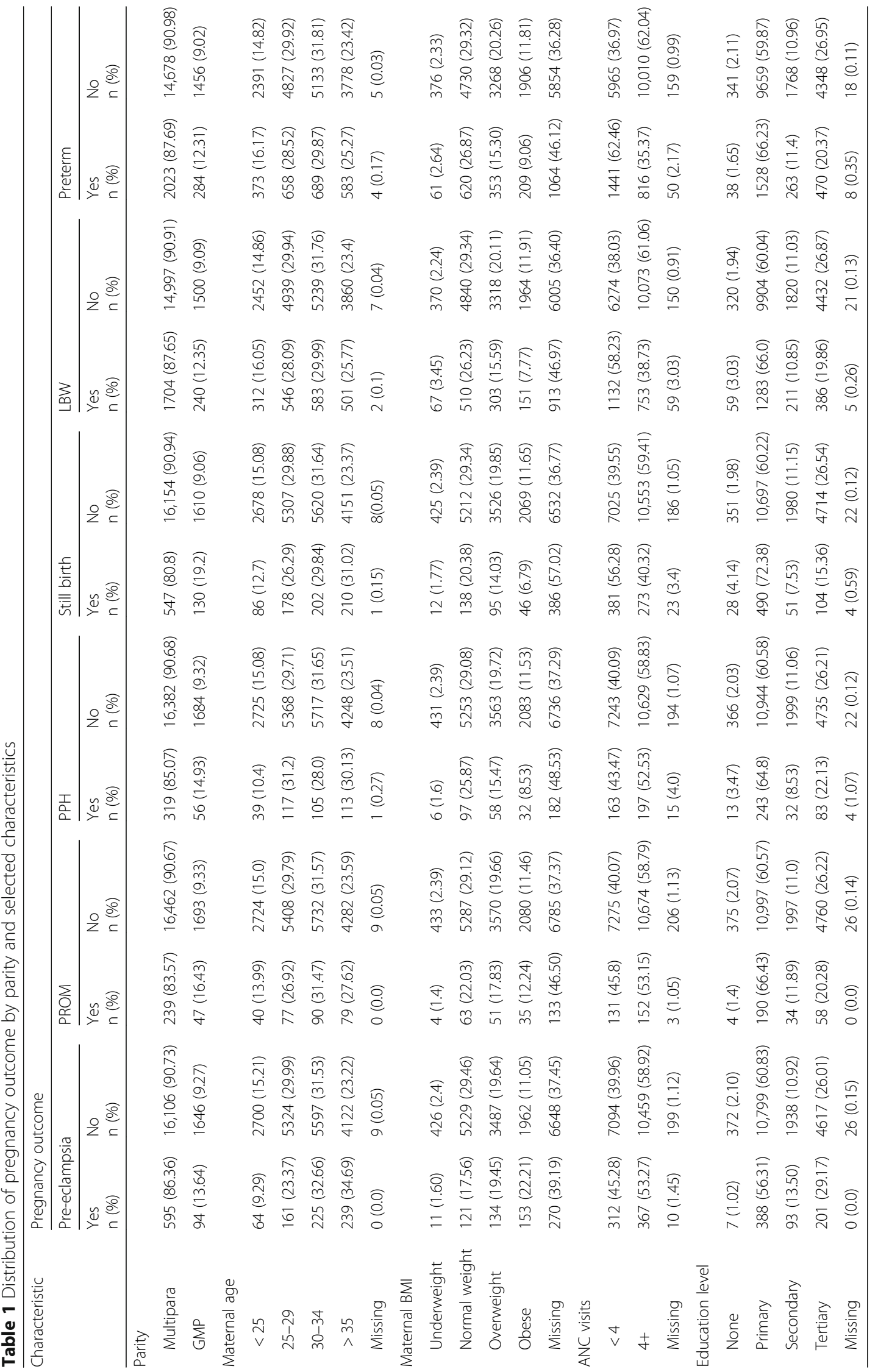


medical birth registry in an electronic database. Information for all deliveries that occur at the department of Obstetrics and Gynecology are prospectively collected and entered in a computerized database system at the medical birth registry. Trained midwife nurses conduct interviews on daily basis using a standardized questionnaire for all women who deliver at the hospital within $24 \mathrm{~h}$ of delivery or immediately after recovery from a complicated delivery. Information on the neonates who are admitted in the neonatal care unit were recorded in neonatal registry forms. Apart from birth registry, each pregnant woman attending this facility has her history documented on patient file with unique file number. These files are confidential and contain all patients' medical records stored at the hospital medical record department. Other patients' information is entered and kept in various registers at each service delivery points such as theater rooms, labor ward, Intensive Care Unit (ICU) and in the antenatal and postnatal units. Data extraction sheet was used to obtain information from demographic characteristics and immediate maternal and fetal complications.

\section{Definition of variables}

The main outcome variables include prelabour rupture of membranes (PROM), postpartum hemorrhage (PPH), pre-eclampsia, low birth weight, stillbirth and preterm birth. Still birth was defined as fetal death of $\geq 28$ weeks of gestation age while preterm birth was defined as babies born alivebefore 37 weeks of pregnancy are completed. Low birth weight (LBW) was defined as a birth weight of less than $2500 \mathrm{~g}$ with gestational age of $\geq 28$ weeks. Independent variables included parity status as the main exposure whereby multiparity was defined as a parity of 2 to 4 deliveries while grand multiparity was defined as a parity of 5 or more deliveries. Maternal socio-demographic characteristics included maternal age, residence, level of education, religion, number of antenatal care ANC-visits, gestational age, body mass index (BMI) and marital status.

\section{Data analysis}

Data analysis was performed using STATA 12.0 (StataCorp LP, College Station, Texas 77,845 USA). After data extraction from the main database, data were cleaned, checked for consistency and verification of missing values. Descriptive statistics were summarized using frequency and percentages for categorical variables. Trend in grand-multiparity was determined by calculating yearly proportions and displayed using a line graph. Chisquare test was used to determine the association between a set of maternal pregnancy outcomes and GMP. Odds ratio (OR) and 95\% confidence interval (CI) for adverse maternal and fetal outcomes associated with GMP were estimated using multivariable logistic regression models. A $p$-value of less than 0.05 (2-tailed) was considered statistically significant.

\section{Results \\ Demographic and obstetrics characteristics of the study population}

A total of 18,441 deliveries were analyzed. Prevalence of grand multiparity was $9.44 \%$. The chi-square test for linear trend showed that, there appears to be a statistically significant trend in prevalence of grand multiparity over years $(p<0.001)$. Demographic and obstetrics characteristics of the study participants are summarized in Table 1. The mean age of women in the study group was 30.4 $(\mathrm{SD}=5.6)$ years. About $64 \%$ of grand multiparous women in this study were aged $>35$ years and more than half $(62.5 \%)$ resided in rural area of North-Tanzania. Approximately half $(52 \%)$ of the grand multiparous subjects had less than 4 antenatal care visits during their pregnancy while $60 \%$ delivered at term.

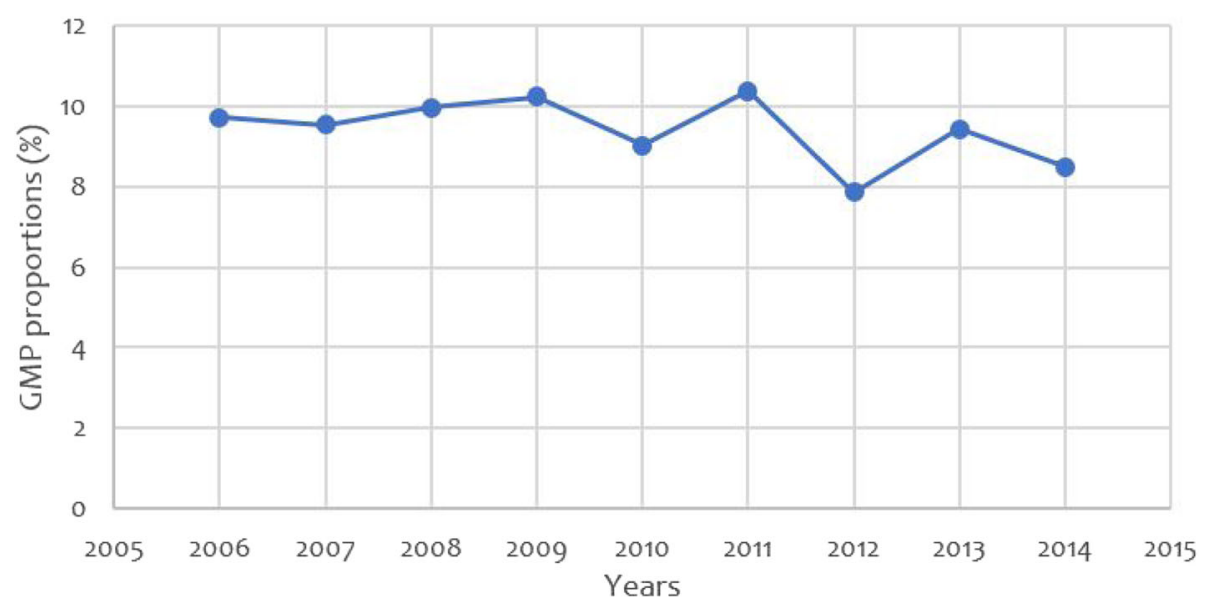

Fig. 2 Trend of grand multiparity from 2006 to2014 at KCMC 


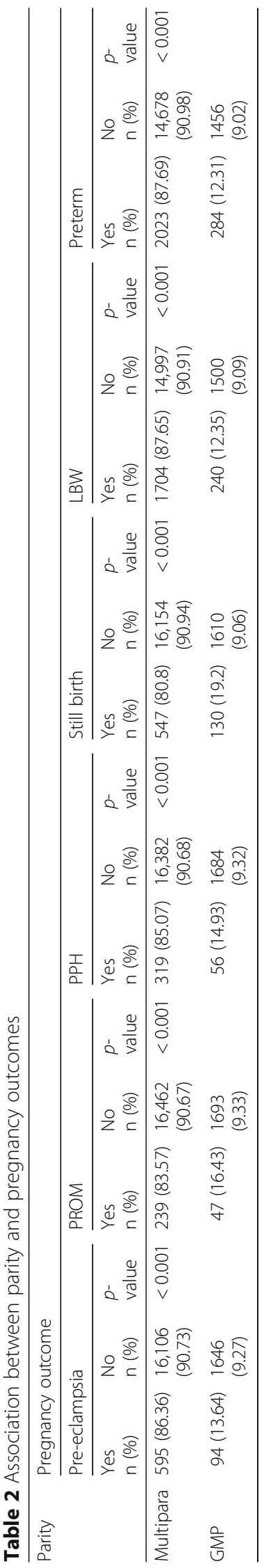




\section{Trend of grand multiparity}

Trend of GMP among women who delivered at KCMC from 2006 to 2014 was seen to decrease over years. Although there is variation in each year, the proportion in the year 2006 (9.72\%) and 2007 (9.03\%) are rather similar. The highest proportion was appreciated in the year $2011(10.38 \%)$ followed by $7.85,9.43$ and $8.49 \%$ in the years 2012, 2013 and 2014 respectively (Fig. 2).

\section{Association between pregnancy outcomes and grand multiparity}

The Chi-square test for pregnancy outcomes and parity is shown in Table 2. In this test, all pregnancy outcomes assessed in the study were significantly associated with parity status of participants.

The crude and adjusted analysis for the association between grand multiparity and adverse pregnancy outcomes are displayed in Table 3 and Table 4 respectively. After adjusting for maternal age, maternal education, mothers' residence, body mass index (BMI), number of antenatal care visits and gestational age, PROM (AOR 1.78; 95\% CI: $1.28-2.49$ ) remained significantly associated with grand multiparity. When fetal outcomes were adjusted for the same factors, stillbirth (AOR 1.66; 95\% CI: $1.31-2.11$ ) and preterm birth (AOR 1.28; 95\% CI: $1.05-1.56)$ remained statistically significant factors associated with GMP (Table 4).

\section{Discussion}

The prevalence of grand multiparity at the institution over nine years found was found to be $9.44 \%$. This study found that grand multiparity was associated with maternal and perinatal complications such as prelabour rupture of membranes, stillbirth and preterm birth. The prevalence of grand multiparity in this study was consistent with a study done in Saudia by Alsammamiet al which reported a prevalence of 5.3\% [15]. However, this is slightly lower compared to other studies which reported the high prevalence of 10.2 and $26.5 \%$ respectively $[9,13]$. The higher prevalence in the later studies could be explained by high prevalence of young marriage and poor acceptance of modern family planning methods. The lower prevalence in this study can be attributed to standard literacy level of grand multiparas, as over $52 \%$ had at least primary level of education.
In the present study, we found that the trend of grand multiparity to be decreasing over nine years.. This decline could have a broader range of attributes including availability of higher education to women and increased community awareness on the health risks of giving birth at an advanced maternal age. The study site has better schools and universities that provides with an opportunity to learn and become aware of reproductive health status. However, the first five (5) years in the trend show no bigger differences while in the year 2011 the rise is seen and then followed by the decline. This can be explained by the fact that at this particular time, the country had adapted well to the strategic plan developed in 2008 known as One Plan which aimed to provide guidance and improve reproductive, maternal, newborn and child health [16].

In this study we found a significant increased risk of prelabour rupture of membranes (PROM) in grand multiparous women compared to those with low parity. Our result is consistent with previous studies done in Nigeria that describe a $3.6-4.2 \%$ increase in PROM in grand multiparas as compared to multiparas [16, 17]. Advanced maternal age (AMA) has been reported as a major risk factor in developing other maternal conditions including hypertension, diabetes mellitus, renal diseases, and other chronic infections which in turn are associated with PROM $[18,19]$. This could be the case in the present study.

In multivariable regression analyses, our study did not find a significant association between PPH and GMP. This is in consistent with study done by Selo-Ojemeet al among Nigerian women [21]. Similar findings were reported by Combs et al who studied women from the United States [22]. We also found that GMP was significantly associated with an increased risk of PPH when controlled by maternal age and educational level attainment. However, addition of other covariates in the model led to non-significant association. Hence, it may suffice to say that, in this population, $\mathrm{PPH}$ is well explained by GMP condition, maternal age and education level. Due to increased maternal age and repeated deliveries, the uterine wall becomes weak resulting in inability to adequately contract leading to development of $\mathrm{PPH}$ [22]. However, in contrast, several other studies elsewhere have clearly shown GMP to be a risk factor

Table 3 Model 1: Unadjusted effects of parity on pregnancy outcome

\begin{tabular}{|c|c|c|c|c|c|c|c|c|c|c|c|c|}
\hline \multirow[t]{3}{*}{ Parity } & \multicolumn{12}{|c|}{ Pregnancy outcome } \\
\hline & \multicolumn{2}{|c|}{ Pre-eclampsia } & \multicolumn{2}{|c|}{ PROM } & \multicolumn{2}{|l|}{$\mathrm{PPH}$} & \multicolumn{2}{|c|}{ Still birth } & \multicolumn{2}{|l|}{$\underline{\text { LBW }}$} & \multicolumn{2}{|c|}{ Preterm } \\
\hline & COR & $95 \% \mathrm{Cl}$ & COR & $95 \% \mathrm{Cl}$ & COR & $95 \% \mathrm{Cl}$ & COR & $95 \% \mathrm{Cl}$ & COR & $95 \% \mathrm{Cl}$ & COR & $95 \% \mathrm{Cl}$ \\
\hline Multipara $^{\mathrm{RC}}$ & 1 & & 1 & & 1 & & 1 & & 1 & & 1 & \\
\hline GMP & 1.55 & $1.24-1.93$ & 1.91 & $1.39-2.62$ & 1.71 & $1.28-2.28$ & 2.38 & $1.95-2.91$ & 1.41 & $1.22-1.63$ & 1.42 & $1.24-1.62$ \\
\hline
\end{tabular}

Note: RC (Reference category) 
Table 4 Model 2: Adjusted effect of parity on pregnancy outcome

\begin{tabular}{|c|c|c|c|c|c|c|c|c|c|c|c|c|}
\hline \multirow[t]{3}{*}{ Parity } & \multicolumn{12}{|c|}{ Pregnancy outcome } \\
\hline & \multicolumn{2}{|c|}{ Pre-eclampsia } & \multicolumn{2}{|c|}{ PROM } & \multicolumn{2}{|l|}{$\mathrm{PPH}$} & \multicolumn{2}{|c|}{ Still birth } & \multicolumn{2}{|l|}{ LBW } & \multicolumn{2}{|c|}{ Preterm } \\
\hline & $\mathrm{AOR}$ & $95 \% \mathrm{Cl}$ & $\overline{A O R}$ & $95 \% \mathrm{Cl}$ & $\mathrm{AOR}$ & $95 \% \mathrm{Cl}$ & $\overline{A O R}$ & $95 \% \mathrm{Cl}$ & $\overline{A O R}$ & $95 \% \mathrm{Cl}$ & $\mathrm{AOR}$ & $95 \% \mathrm{Cl}$ \\
\hline Multipara $^{\mathrm{RC}}$ & 1 & & 1 & & 1 & & 1 & & 1 & & 1 & \\
\hline GMP & 1.32 & $0.94-1.85$ & 1.78 & $1.28-2.49$ & 1.34 & $0.95-1.9$ & 1.66 & $1.31-2.11$ & 1.26 & $0.97-1.62$ & 1.28 & $1.05-1.56$ \\
\hline
\end{tabular}

Note: RC (Reference category)

for PPH $[17,20,23]$. Few data that didn't demonstrate an increased risk for PPH in grand multiparity could be attributed to a small scale design with inability to capture rare adverse events [13].

This study has demonstrated that GMP women had an increased odds of experiencing stillbirth and preterm births as compared to women with low parity as previously described in published data from Uganda and Nigeria [9, 21, 22]. This could be explained by the fact that since AMA is a major risk for various maternal complications, thus fetus and neonates may always be susceptible to mortality and morbidity. Other fetal complications which have been reported in other studies in Tanzania with inadequately controlled intrapartum confounders include meconium stained liquor and low apgar score which were found to be insignificant complications in the present study.

\section{Strengths and limitations}

This hospital-based study used a birth registry data collected routinely at KCMC using a standardized questionnaire. From this data set a large sample size was obtained thus giving the study more power and making it possible to adjust for confounders as well as making inference in similar settings with high precision. Despite the strengths, there were some limitations that should be considered while interpreting findings from this study. Since this was a referral hospital-based study, our results may not be generalizable to the general population of Tanzania. In attempt to minimize overrepresentation of high-risk women, we excluded women with multiple pregnancy. In addition, maternal mortality, as one of the important outcomes was not investigated in this study due to insufficient information regarding maternal deaths in the dataset. We therefore recommend future prospective study to explore the association of GMP with maternal mortality.

\section{Conclusion}

Despite low prevalence of GMP at the institution, it continues to pose additional challenges to fetal and maternal health. Although the GMP proportion has decreased over years, our result reveals a rather gradual decline of GMP overtime. The study warrants more emphasis to be directed towards community sensitization on the adverse maternal and fetal outcomes that might be attributable to GMP. The study also suggests a need for establishment of a referral system unit at this institution that will be specifically managing deliveries occurring in women with high parity as well as advanced age.

\section{Abbreviations \\ AMA: Advanced maternal age; ANC: Antenatal clinic; AOR: Adjusted odds ratio; BMI: Body mass index; Cl: Confidence interval; COR: Crude odds ratio; GMP: Grand multiparity; ICU: Intensive care unit; KCMC: Kilimanjaro Christian Medical Centre; LBW: Low birth weight; PROM: Prelabour rupture of membranes}

\section{Acknowledgments}

We are grateful to the collaboration between KCMC and the University of Bergen in Norway for the establishment of the Birth registry that has made this work possible. Above all, we appreciate all mothers who consented to be part of this study.

\section{Authors' contributions}

ZM: Designed the study, performed the statistical analysis and participated in writing the manuscript, CST: Data analysis, participated in manuscript writing and reviewed the final manuscript EM: contributed in drafting the

manuscript, MJM: statistical consultation and reviewed the manuscript, BM: participated in designing the study, reviewing of manuscript. All authors have read and approved the final manuscript.

Funding

No funding was obtained for this study.

Availability of data and materials

The datasets during the current study are not publicly available to protect the participants' anonymity. But it can be freely available from the corresponding author on reasonable request.

\section{Ethics approval and consent to participate}

Ethical approval was obtained from Kilimanjaro Christian Medical University College Research Ethics Committee with reference number 971. Permission to use medical birth registry was obtained from KCMC administrative authority. For practical reasons, the midwives give oral information individually to each mother and then asked her willingness to participate/be enrolled in the study and their data to be used for the public benefits. Following consent, the mother could still choose not to reply to single question. In order to document the consent process, the birth registry has an instruction manual for registration of deliveries in the registry. This manual contains the standard operating procedures on how to conduct the interview and also has a description of the basic rules including ethical guidelines such as consent process. The consent procedure was approved by the ethics committee as it was part of the study protocol.

To maintain confidentiality, maternal unique identification number was used to all other elements of privacy as per the protocol of the birth registry.

\section{Consent for publication}

A verbal consent to publish individual details (information) were provided by each of the study participants after being explained about the objectives of the medical birth registry and the benefits of the data generated to be shared to the public. 


\section{Competing interests}

The authors declared to have no competing interests.

\section{Author details}

'Department of Obstetrics and Gynecology, Kilimanjaro Christian Medical Centre, Box 3010, Moshi, Tanzania. ${ }^{2}$ Department of Epidemiology and Biostatistics, Institute of Public Health, Kilimanjaro Christian Medical University College, Box 2240, Moshi, Tanzania. ${ }^{3}$ Dar es Salaam Institute of Technology, Department of Science and Laboratory Technology, Box 2958, Dar es Salaam, Tanzania.

Received: 12 January 2019 Accepted: 24 June 2019

Published online: 02 July 2019

\section{References}

1. Bugg GJ, Atwal GS, Maresh M. Grand multiparae in a modern setting. BJOG An Int J Obstet Gynaecol. 2002;109(3):249-53.

2. Opara El, Zaidi J. The interpretation and clinical application of the word "parity": a survey. BJOG An Int J Obstet Gynaecol. 2007;114(10):1295-7.

3. Babinszki A, Kerenyi T, Torok O, Grazi V, Lapinski RH, Berkowitz RL. Perinatal outcome in grand and great-grand multiparity: effects of parity on obstetric risk factors. Am J Obstet Gynecol. 1999;181(3):669-74.

4. Chandra A, Copen CE, Stephen EH. Infertility and impaired fecundity in the United States, 1982-2010: data from the National Survey of family growth. Natl Health Stat Report. 2013;(67):1-18.

5. Njoku CO, Abeshi SE, Emechebe Cl. Grand Multiparity : obstetric outcome in comparison with multiparous women in a developing country. Open J Obstet Gynecol. 2017:7(7):707-18

6. Eze JN, Okaro JM, Okafor MH. Outcome of pregnancy in the grand multipara in Enugu, Nigeria. Trop J Obstet Gynaecol. 2006;23:8-11 Available from: http://dx.doi.org/10.4314/tjog.v23i1.14555.

7. Kuti O, Dare F, Ogunniyi S. Grand multiparity: mothers' own reasons for the index pregnancy. Trop J Obstet Gynaecol. 2001;18(1):31-3.

8. Solomons B. The dangerous multipara. Lancet. 1934;224(5784):8-11.

9. Hoque M, Hoque E, Kader SB. Pregnancy complications of grand multiparity at a rural setting of South Africa. Iran J Reprod Med. 2008;6(1):25-31.

10. Mutihir J. Obstetric outcomes of the grand multipara in Jos, Nigeria. J Med Trop. 2005;7(1):14-20.

11. Maro EW, Mosha NR, Mahande MJ, Obure J, Masenga G. Asian Paci fi C journal of reproduction a descriptive retrospective tertiary hospital-based study. Asian Pacific J Reprod 2016;5(3):214-220. Available from: https://doi. org/10.1016/j.apjr.2016.04.012

12. Emechebe C, Njoku C, Eyong E, Maduekwe K, Ukaga J. The social class and reasons for grand multiparity in Calabar, Nigeria. Trop J Obstet Gynaecol. 2016;33(3):327 Available from: http://www.tjogonline.com/text.asp?2016/33/ 3/327/199808

13. Ogbe AE, Ogbe BP, Ekwempu C. Obstetric outcome in grand multiparous women in Jos teaching hospital. Jos J Med. 2010;6(2):39-43.

14. Toohey JS, Keegan KA, Morgan MA, Francis J, Task S, deVeciana M. The "dangerous multipara": fact or fiction? Am J Obstet Gynecol. 1995;172(1): 683-6.

15. Alsammani M, Ahmed S. Grand multiparity: risk factors and outcome in a tertiary hospital: a comparative study. Mater Sociomed. 2010;26(3):181-97 Available from: https://www.ncbi.nlm.nih.gov/pmc/articles/PMC4610637/.

16. Ministry of Health Community Development Gender Elderly and Children. The National Road Map Strategic Plan to Improve Reproductive, Maternal, Newborn, Child and Adolescent Health in Tanzania (2016-2020) (One Plan II). 2016. Available from: https://www.globalfinancingfacility.org/sites/gff_ new/files/Tanzania_One_Plan_Il.pdf. Accessed 15 Dec 2018.

17. Idoko P, Nkeng G, Anyawu M. Reasons for current pregnancy amongst grand multiparous Gambian women - a cross sectional survey. BMC Pregnancy Childbirth. 2016;16(1):1-6. Available from. https://doi.org/10.1186/ s12884-016-1016-7.

18. Afolabi AF, Adeyemi AS. Extreme grand multiparity: is it an obstetric risk factor? Eur J Obstet Gynecol Reprod Biol. 2002;101(1):22-5.

19. Minkoff H, Grunebaum AN, Schwarz RH, Feldman J, Cummings M, Crombleholme $W$, et al. Risk factors for prematurity and premature rupture of membranes: a prospective study of the vaginal flora in pregnancy. Am J Obstet Gynecol. 1984;150(8):965-72.
20. Ananth CV, Oyelese $Y$, Srinivas N, Yeo L, Vintzileos AM. Preterm premature rupture of membranes, intrauterine infection, and oligohydramnios: risk factors for placental abruption. Obstet Gynecol. 2004;104(1):71-7.

21. Okonofua FE. Gynecology and obstetrics risk factors for primary postpartum haemorrhage. Arch Gynecol Obstet. 1997;259:179-87.

22. Combs CA, Murphy EL, Laros RK. Factors associated with postpartum hemorrhage with vaginal birth. Obstet Gynaecol. 1991;77(1):69-76.

23. Drife J. Management of primary postpartum haemorrhage. BJOG An Int J Obstet Gynaecol. 1997;104(3):275-7.

\section{Publisher's Note}

Springer Nature remains neutral with regard to jurisdictional claims in published maps and institutional affiliations.
Ready to submit your research? Choose BMC and benefit from:

- fast, convenient online submission

- thorough peer review by experienced researchers in your field

- rapid publication on acceptance

- support for research data, including large and complex data types

- gold Open Access which fosters wider collaboration and increased citations

- maximum visibility for your research: over $100 \mathrm{M}$ website views per year

At BMC, research is always in progress.

Learn more biomedcentral.com/submissions 